\title{
The Psycho-Neuro-Endocrino-Immunology (Pnei) of the Cardiovascular System
}

\author{
Paolo Lissoni, Arianna Lissoni, Francesco Pelizzoni*, Franco Rovelli, Roberto Trampetti and Giuseppe Di Fede \\ Istituto di Medicina Biologica, Milan, Italy
}

Submission: September 23, 2019; Published: October 30, 2019

*Corresponding author: Francesco Pelizzoni, Divisione di Cardiochirurgia, Ospedale Niguarda, Milano, Italy

\begin{abstract}
The immune system has appeared to regulate not only the immune response, but all biological functions, including the nervous, the endocrine and the cardiovascular systems. At the other side, the immune system is under a physiological psycho neuroendocrine regulation, mainly modulated by the pineal gland and the opioid system, respectively in a stimulatory or in a suppressive way by influencing the cytokine network. The different cytokines exert different immunobiological effects on the immune cells, mainly by influencing the interactions between lymphocyte and monocyte-macrophage systems. The functionless of the whole immune system may be synthesized by the lymphocyte-to-monocyte ratio (LMR), and the evidence of an abnormally low LMR has been proven to be associated with a poor prognosis in all systemic severe inflammationrelated human diseases, including cancer, autoimmune diseases and the cardiovascular disorders.

Within the cytokine group, IL-17, IL-6, IL-1 beta, TNF alpha, and IL-18 represent the most important inflammatory cytokines involved in systemic human pathologies, whereas the most effective anti-inflammatory cytokines are consisting of TGF-beta and IL-10. The evidence of abnormally high levels of inflammatory cytokines may predict a worse prognosis in all systemic diseases. Within the neuroendocrine system, the secretion of melatonin (MLT), which represents the most investigated immunomodulating pineal hormone, has appeared to increase LMR by stimulating lymphocyte proliferation and inhibiting the monocyte macrophage system. Moreover, it has been shown that a decrease in MLT secretion may be associated with a poor prognosis in cardiovascular diseases. Therefore, MLT administration could be effective in reducing myocardial infarction and stroke-induced tissue damage. Further studies, by concomitantly evaluating MLT secretion in relation to changes in LMR values, as well as in IL-17 and IL-18 blood levels, will be required to establish whether the immune changes occurring during cardiovascular and systemic inflammatory diseases may depend at least in part on a diminished pineal function.
\end{abstract}

Keywords: Autoimmunity; Cancer, Cannabinoids; Cardiac ischemic disease; IL-2; IL-12; IL-17; IL-18; Lymphocyte-to-monocyte ratio; Melatonin; Opioid system; Pineal gland

\section{Introduction}

\section{The Olistic Function of the Immune System}

The recent advances in the knowledge of cytokine network have demonstrated that the overall biological functions and systems, including the cardiovascular one, are physiologically under a regulatory control played by the immune system through the release of cytokines from the activated immune cells. It has been shown that several inflammatory cytokines, including IL-6, IL-2 and TNF-alpha, may induce hypotension by allowing both nitric oxide (NO)-related vasodilatator effect and enhanced vascular permeability, with a following fluid loss in the interstitial space [1]. Moreover, the immune system would play a fundamental role in the control not only of the immune reactions, but the overall biological systems, namely the nervous, the endocrine, the haematopoietic and the cardiovascular systems. In fact, the cytokines released by the activated immune cells may induce both local and systemic effects by exerting endocrine-like activities.

From an immunological point of view,, despite the great complexity of immune cells involved in regulating the immune responses, the overall immune reactions may be synthetically considered as the end-result of the interactions among three essential immune cells, consisting of lymphocytes, dendritic cells (DCs) and monocyte-macrophage cells, with the DCs as the link between lymphocyte and monocyte systems [2]. In more detail, the immune responses would essentially depend on the interactions between lymphocyte and monocyte functions, and most severe human diseases, including cancer, autoimmune diseases and cardiovascular disorders would substantially be characterized by an altered balance between lymphocyte and monocytes functions, with a decline in lymphocyte count in association with a concomitant increase in monocyte count and 
activity, with a following decline in lymphocyte-to-monocyte ratio (LMR). In fact, the evidence of abnormally low values of LMR has been proven to predict a poor prognosis in all severe human diseases, including cancer autoimmune diseases and cardiovascular pathologies [3-5].

\section{Clinical significance of LMR}

The LMR Is a systemic inflammatory marker, very simple and inexpensive, for prediction of disease onset, progression and survival. In normal conditions, LMR must be at least greater than 2. In other words, lymphocyte count must be at last two times greater than that of monocytes. The decline in LMR values is associated with a negative prognosis in most human pathologies, including cancer, autoimmunity and cardiovascular pathology, even though the mechanisms involved in LMR decrease may be different. In fact, in the neoplastic pathologies the decline in LMR would be mainly due to an important decline in lymphocyte count, with may be similar to that occurring in AIDS patients [6], whereas it would mainly depend on an increase in monocyte count in both autoimmunity and cardiovascular diseases which only rarely may present a severe lymphocytopenia.

Then, the LMR may be considered as a marker of the biological response, by confirming the importance of host immunobiological response in influencing the clinical course of human severe diseases. The clinical and biological values of LMR is justified by the evidence that circulating monocyte count have been proven to be positively correlated with the functional activity of the macrophage system, including macrophage tumor infiltration [7]. The different functions and subsets of $\mathrm{T}$ and $\mathrm{B}$ lymphocytes have been well defined, particularly that of CD4+ $\mathrm{T}$ lymphocytes, which consist of three major sub-populations [8-10], including T helper-1 (TH1) lymphocytes (CD4+CD25CD17-), that activates the whole immune cells by releasing $\mathrm{LL}-2$ and IFN-gamma, regulatory T lymphocytes (T reg) (CD4+CD25+), which in contrast play an immunosuppressive activity through the secretion of TGF-beta and IL-10, and TH-17 lymphocytes (TH17) (CD4+CD17+), that may inhibit $\mathrm{T}$ reg system and induce the inflammatory response by stimulating both macrophages and TH1 cells. On the contrary, both monocytes and macrophage are less characterized and classified in different manners, and there is no agreement in relation to their classification.

\section{Monocyte and macrophage classification}

Despite some controversial results, macrophages are generally subdivided into two essential subpopulations, consisting of M1 and M2-macrophages [11]. M1-macrophages are commonly considered to play an inflammatory activity, whereas M2-macrophages would exert a major anti-inflammatory action. Then, M1- cells may induce tissue damage, whereas M2 cells would have a repairing activity. M1 cells stimulates TH1 differentiation. In contrast, M2 cells would promote TH2 cell differentiation. At the other side, TH1 may induce Ml cell activation by secreting IFN-gamma, whereas TH2 cells would stimulate M2 cell functions through the release of IL-4 and IL-13. Moreover, TGF-beta would be essential for M2 cell activation. M1macrophages mainly produce NO, whereas, which is provided by an inhibitory action on cell proliferation.

In contrast, M2-macrophages produce ornithine instead of NO. In vitro, M1-macrophages is namely induced by GM-CSF, while M2-macrophages are mainly stimulated by M-CSF. M1macrophages are also stimulated by 17 -beta-estradio, and this evidence could explain some differences in the immune functions occurring between men and women. In any case, the major difference between M1 and M2 macrophages would regard the different cytokine production. M1 macrophages namely secrete inflammatory cytokines, including TNF-alpha, IL-6, IL-8, IL-12, IL-23, and IL-1 beta. On the contrary, M2 macrophages namely produce anti-inflammatory immunosuppressive cytokines, including TGF-beta and IL 10, in less amounts IL-1 beta as inflammatory cytokine, and IFN-alpha and beta.

As far as the action on cancer growth is concerned, despite the inflammatory response may play a stimulatory action on cancer growth [12], M1-macrophages are generally considered to be anti-tumor cells because of their capacity of killing normal and tumor cells, as well as interacting with TH1 lymphocytes, since $M$ cell deficiency has appeared to allow a diminished TH1-dependent antitumor immune response. in contrast, M2macrophages would play a major pro-tumoral role, due to their production of immunosuppressive cytokines, namely TGF-beta, which in turn stimulates M2 cell differentiation, angiogenic factors, namely VEGF, and tumor growth factors, such EGF. In addition, M2 macrophages would promote the generation of T reg lymphocytes, which suppress TH1-, DC- and cytotoxic T cell dependent anticancer immunity [13].

Based on their activity, M1-macrophages are also called as Fight macrophages because of their capacity of killing cells, and M2-macrophages are also defined as Fix macrophages because of their ability to repair tissue damage. On the basis on their response to various agents, M2-macrophages would be further subdivided into three may subsets, consisting of M2a, M2b and M2c- macrophages. IL-4 would may promote M2a cell generation, immunoglobulins that of M2b cells, while TGF-beta and IL-10 themselves would stimulate M2c differentiation. Macrophage subsets may be also differentiated based on their expression of CD molecules. Both macrophages are CD68+ cells, but Ml cells are namely CD80+, while M2 cells are namely CD163+ cells.

Moreover, the expression of CD14 and CD16 would be greater in M2 macrophages. According to their different and apparently apposite function, M1-to-M2 ratio would play an important role in the regulation of the immune responses. In any case, $\mathrm{Ml}$ and M2 macrophages would represent different functions rather than different cell populations, with their possible transformation in a bidirectional manner, according to the so-called macrophage plasticity $[11,14]$. As far as monocyte classification is concerned 
[15], monocytes are generally subdivided into three subsets, all characterized by the expression of CD14, but with differences in that of CD16, which is absent in the so-called classical monocytes (CM) (CD14+CD16-), intermediate in the so-called intermediate monocytes (IM) (CD14++CD16+), and high in the non-classical monocytes (NCM) (CD14+CD16++).

CD14 Is a toil-like receptor, while CD16 Is the receptor for the Fc fragment of the immunoglobulins. CM cells would represent about $90 \%$ of all monocytes, while the remaining ceils are consisting of IM and NCM cells. CM cells play a major pro-inflammatory action, whereas NCM would exert an antiinflammatory activity. The role of IM cells is still controversial, since in some experimental and clinical conditions they have appeared to exert an inflammatory action and a tissue damage superior to those played by CM cells themselves [16]. This evidence may be explained on the basis that IM cells would represent the monocyte subset most active in the production of inflammatory cytokines, which are responsible for tissue damage. CM cells may present a high expression of chemokine receptor far CCR2 and low for CX3CR1, whereas an opposite condition is presented by IM cells. Then, NCM cells are the only monocyte subset provided by anti-inflammatory activity through the production of anti-inflammatory cytokine, such IL-10, which, however, plays an immunosuppressive effect on the anticancer immunity by stimulating $\mathrm{T}$ reg cell generation.

\section{Monocyte-Macrophage Function in Cancer, Autoimmunity and Cardiovascular Diseases}

\section{Macrophage-Monocyte System in Cancer}

The macrophage-mediated chronic inflammatory response has appeared to stimulate cancer growth, by either producing immunosuppressive cytokines, or angiogenic or tumor growth factors. Irrespectively of macrophage subtypes, tumor macrophage infiltration stimulate cancer growth, as well that of $\mathrm{T}$ reg cells, whereas TH1 and cytotoxic T cells inhibit cancer cell proliferation and dissemination [16]. Lymphocytopenia is the main clinical biomarker of cancer progression, while the increase in monocyte count would characterize the only disseminated phase of the neoplastic disease. Moreover, advanced cancer-related lymphocytopenia is mainly due to a decline in TH1 lymphocytes, in association with an increased T reg cell percentage. According to LMR values, the immune status of cancer patients may be simply classified in an easy way in three different prognostics conditions [17]: 1) good prognosis: normal values of LMR higher than 2.1 with a concomitant lymphocyte count greater than $1,500 / \mathrm{mm} 3 ; 2$ ) intermediate prognosis: LMR within the normal range, but with lymphocyte number less than $1000 / \mathrm{mm} 3$; 3 ) poor prognosis: LMR less than 2.1 with low lymphocyte count. On the contrary, the evaluation of the different monocyte subsets has been less investigated, since most studies have been performed to analysed the immune status at tumor micro-environment.

\section{Macrophage-Monocyte system in Autoimmunity and Cardiovascular Diseases}

With respect to cancer, both autoimmune diseases and cardiovascular diseases, including the cardiac ischemic disease (CID) are more characterized by an early intervention of monocyte-macrophage system in determining a more severe clinical course of the disease [18]. In more detail, autoimmune diseases mainly due to auto-antibody production, including namely systemic lupus erythematosus (SLE), as well as rheumatoid arthritis (RA), would be due to an heritance activation of M2-macrophages, whereas those namely due to a cellular-mediated autoimmunity, including multiple sclerosis (MS) and inflammatory bowel disease (IBD), would mainly depend on an enhanced M1-macrophage activation [19]. Therefore, macrophage polarization would influence the clinical course of the autoimmune disease, depending on Ml to-M2 ratio.

By summarizing, autoimmune diseases may be exacerbated by M1 macrophage activation and controlled by M2 macrophage stimulation. Neurodegenerative disease would be mainly due to M1 macrophage enhanced activation, while allergy seem to be mainly related to an enhanced M2 cell activity. At the other side, the main cells responsible for progression of atherosclerosis would consist of Ml macrophages [20]. As far as monocyte subtypes in autoimmunity, even though there are controversial results, it would seem the exacerbation of disease is mainly due to an increase in CM cell count, particularity in SLE, which is generally associated with a concomitant deficiency in IM cells. In contrast, NCM cell count is generally with the normal range in autoimmune diseases, even though their inhibitory action on DC activation and lymphocyte proliferation would be diminished.

\section{Monocyte Subsets and Functions in Cardiovascular Diseases}

Monocytes have been proven to play an important pathogenetic role in both CID and atherosclerosis. In fact, it has been demonstrated that monocytes may promote the progression of atherosclerosis through infiltrating the arterial wall and promoting the inflammatory response [20]. An enhanced monocyte activation has been proven to allow to a worse prognosis in both myocardial infarction by counteracting the occurrence of reflow phenomenon, and atherosclerosis by inducing a chronic inflammatory status [21]. Lymphocytes also have appeared to be involved in the heart ischemic diseases, and in particular it has been shown that the infiltration of the ischemic myocardial area by regulatory $\mathrm{T}$ lymphocytes ( $\mathrm{T}$ reg) may allow a better prognosis in the case of myocardial infarction by promoting wound healing and remodelling after myocardial infarction, whereas $\mathrm{T}$ helper-1 (TH1) infiltration would amplify the myocardial damage by promoting the inflammatory response. The enhanced monocyte-macrophage function during atherosclerosis and myocardial infarction may simply be clinically documented by the evidence of abnormality 
low values of lymphocyte-to-monocyte ratio (LMR), which could constitute a simple and inexpensive biomarker to monitor the clinical response during the myocardial infarction and other cardiovascular diseases.

\section{Cytokine Network and Lymphocyte-to-Monocyte Ratio}

The values of LMR would represent the end-result of cytokine network functionless, particularly of the interactions between pro-inflammatory and anti-inflammatory cytokines. Therefore, to better define its clinical and physio-pathological significance in the single patient, LMR would have to be related to the blood levels of the main cytokines involved in the stimulation of lymphocyte and monocyte proliferation and activation, by taking into consideration that $\mathrm{T}$ lymphocyte proliferation is mainly determined by IL-2 [8], while the differentiation into TH1 cells is mainly stimulated by IL-12 and inhibited by IL-4. Monocyte proliferation is mainly induced by GM-CSF and M-CSF, while the differentiation into $\mathrm{Ml}$ or M2 subtypes is namely determined by IFM-gamma and TGF-beta plus IL-4, respectively. Then, an increased secretion of inflammatory cytokines, namely IL-6, IL-l beta and TNF-alpha would allow a decline in LMR values, whereas the prevalence of anti-inflammatory cytokines may determine an increase in LMR values.

\section{Cytokine Network in Cancer}

The progression of cancer is mainly characterized by a constant decline in blood levels of those cytokine involved in lymphocyte differentiation and activation, including IL-2 and IL-12 [22], in association with an increase in the concentrations of inflammatory cytokines, namely IL-6, TNF-alpha and IL-1 beta, and/or anti-inflammatory immunosuppressive cytokines, including TGF-beta and IL-10, being the anti-inflammatory action linked to the immunosuppressive one. Then, cancerrelated lymphocytopenia is associated with low blood levels of IL-2 and IL-12, and high concentrations of TGF-beta, TNF-alpha and IL-6, which in contrast stimulate the macrophage-monocyte system.

\section{Cytokine Network in the Autoimmunity}

The auto-immune disease are characterized by a general enhanced production of inflammatory cytokines, including IL6, IL-i beta and TNF-alpha, with some preferential profiles in relation to the different diseases, such as the evidence of high levels of TNF-alpha particularly in the RA, while the cytokine profile in SLE is still more controversial. Moreover, recent observations have confirmed the fundamental role of IL-17 in the pathogenesis of the autoimmune diseases, being responsible for the induction and the maintenance of the inflammatory status [10] because of the ability of IL-17 to activate both TH1 cell and macrophage functions, as well as to inhibit T reg cells, which play an essential role in inhibiting the activation of autoreactive lymphocytes [13].
In any case, the evidence of high levels of anti-inflammatory immunosuppressive cytokines, including TGF-beta and IL-10, is associated with a more favourable clinical course, whereas a decline in IL-10 and TGF-beta production would characterize the active phase of disease, whereas that of high levels of TGF-beta and IL-10 may predict a disease control [23]. More controversial is the secretion of IL- 2 and iL- 12 in the pathogenesis of the autoimmune diseases. Either low or high IL-2 blood concentrations have been described in the autoimmunity. These controversial results may be explained by taking into consideration that IL- 2 may either promote or counteract the autoimmune processes [24] because of its concomitant stimulatory effects on TH1, macrophages and $\mathrm{T}$ reg lymphocytes, and the inhibitory one on TH17 cells.

The inhibition of TH17 cells and the stimulation of T reg lymphocytes could allow a clinical control of the autoimmune process, whereas the stimulatory action on TH1 cell and macrophage activation may exacerbate the autoimmune disease. On the same way, the role of $1 \mathrm{~L}-12$ in the autoimmunity is still controversial. The pro-inflammatory activity of $1 \mathrm{~L}-12$ and its inhibitory effect on $\mathrm{T}$ reg cells could promote the autoimmune reactions, which in contrast could be blocked by its concomitant inhibitory action on iL-17 secretion [25]. Finally, because of its inhibitory effect on M1 macrophage activity, IL-4 could also be provided by therapeutic effects in the autoimmune diseases.

\section{Cytokine Network in Cardiovascular Diseases}

As well as in the autoimmunity, lL-17 and TGF-beta would represent the most important cytokines in influencing the clinical course of the cardiovascular disorders, including CID and atherosclerosis, which may be promoted by IL-17 and controlled by TGF-beta through a modulation of monocyte-macrophage system, which is responsible for the infiltration of the arterial wall in the atherosclerosis and for inflammatory response-induced enhanced tissue damage in the cardiac ischemia and myocardial infarction $[16,21]$. Then monocytes would play an important role in the pathogenesis of atherosclerosis by infiltrating the arterial wall and promoting the inflammatory response and the fibrotic process [20]. At the other side, monocyte rise in CID patients seems to be mainly due to an increase in IM cells (CD14++CD16+) during the whole period following the myocardial infarction in association with normal values of NCM cells (CD14+CD16++), while an increase in CM cells (CD14+CD16-) has been observed only during the first two days after the acute event [21].

Other authors have also described a concomitant increase not only in IM, but also in NCM cells, but its role is still contradictory depending on their cytokine production. On the contrary, TGFbeta, as well as IL-10, may reduce tissue damage by counteracting the infarction-induced inflammatory response, which in contrast enhances tissue damage by inhibiting its repairing processes. In any case, the evidence of an increased percentage of IM cells has been shown to be associated with a poor prognosis in both cerebral and cardiac ischemia [26]. Lymphocytes have been shown to be also involved in influencing the prognosis of CID, 
since the infiltration of the ischemic myocardial area by T reg cells may allow a better prognosis in myocardial infarction through a stimulation of tissue healing and remodelling after myocardial infarction, whereas TH1 infiltration would further worse the prognosis by amplifying the inflammatory response [16]. Finally, it has been recently observed that the concomitant increase in IL-18 level, which may be produced by the same endothelial cells, in association with enhanced IL-17 blood concentrations would further worse the prognosis of CID [26]. Therefore, further studies, by concomitantly evaluating lymphocyte and monocyte subsets in relation to the secretion of the most important proinflammatory and anti-inflammatory cytokines, will be required to better define the functionless of the cytokine network in the cardiovascular diseases.

\section{The Neuroendocrine Regulation of Lymphocyte and Monocyte Functions}

The recent discoveries in the area of psycho-neuroendocrineimmunology (PNEI) have clearly confirmed that both lymphocyte and monocyte-macrophage system are under a neuroendocrine regulation [27]. It has been shown that chronic stress may inhibit M1-macrophage and stimulate M2-macrophage system, with a following decline in M1-to-M2 ratio. M2-macrophages may exert both anti-inflammatory and immune suppressive effects. Then, in most clinical conditions, the main problem is to pilot the M2 cell functional in a preferential immunosuppressive or antiinflammatory action, since M2-macrophages may potentially exert both functions. M2 macrophage increase and activation may reduce the inflammatory response, then induce some benefits in the treatment of autoimmune and cardiovascular diseases, but on the same way their immunosuppressive effects on the antitumor immunity may predispose to cancer and stimulate cancer growth.

At present, the knowledge of the neuroendocrine control of monocyte macrophage system is less defined with respect to that the neuro-immuno-modulation of the lymphocyte system. In fact, it is known that lymphocyte system is namely mediated by two major brain neuroendocrine functional systems, consisting of brain cannabinergic system-pineal gland unit and brain opioid system hypothalamus-pituitary-adrenal axis. The cannabinergicpineal unit is involved in pleasure perception and spiritual expansion of consciousness and from an immunomodulatory point of view in the stimulation of lymphocyte-dependent immune functions and inhibition of macrophage-mediated chronic inflammatory response by activating TH1 and DC functions, with a following enhanced endogenous production of both IL-2 and IL-12 secretions, and inhibiting of TNF-alpha, IL-6, IL-1 beta and IL-17 activities [28,29] whereas the opioidpituitary-adrenal axis, which is activated in stress and depressive conditions, may inhibit TH1 cell and DC functions and stimulate $\mathrm{T}$ reg lymphocyte system, with a following decline in IL-2 and IL-12 levels, and an increase in those of TGF-beta and IL-10 [30].
Stress promotion of human disease would be mainly mediated by an enhanced brain opioid activity, since in experimental conditions the concomitant administration of the mu-opioid antagonist naltrexone has been proven to counteract stress-induced tumor development [31]. A fundamental role in the neuroimmunomodulation is played by the pineal gland, even though most studies have been limited to the investigation of its most known hormone, melatonin (MLT)[32], which has been shown to modulate in vivo the whole cytokine network by enhancing IL-2 and IL-12 secretions and inhibiting those o TNF-alpha, IL-1 beta and IL-6. Moreover, MLT would be also able to counteract TH17 lymphocyte activation [33] and inhibit IL18-induced inflammatory response with modulatory effect on TGF-beta production depending on the different experimental conditions. Fin the evidence of a diminished secretion of MLT with a lack of its physiological light/dark rhythm has appeared to predict a poor prognosis in both cancer and cardiovascular disorders [34-36]. Because of its stimulatory role on Tymphocyte generation and functions, and its inhibitory action on monocyte macrophage system, MLT administration may allow an increase in LMR values. Not only, but MLT has been also appeared to directly modulate monocyte differentiation, and it has been shown that monocyte evolution into macrophages is associated with a progressive decline in MLT receptor expression [37].

If the knowledge of the only biological effects of MLT, which simply represents the most investigated pineal hormone, has already contributed to reinterpretation the most severe human diseases, including the cardiovascular ones, according to an neuro-endocrino-immunological point of view, more surprising discoveries could be achieved in understanding the influence of the psycho-spiritual life on the biological body when the whole pineal biochemistry will be investigated, in particular the action of pinealine and 5-methoxytryptamine, which have been already proven to exert immunostimulatory, anti-inflammatory, and anticancer activities [38]. Within the cytokine network, from a physiopathological point of view it would be particularly fundamental to control IL-17 secretion, which has been proven to plays a negative prognostic significance in human severe diseases, including cancer, autoimmunity and cardiovascular diseases [39].

Then, the inhibition of IL-17 secretion and activity could exert important therapeutic implications. At present, it is already known that IL-17 secretion may by inhibited by cannabinoids MLT itself at least in some conditions and IL-12 [25,33]. The decline in IL-17 blood concentrations could have important therapeutic effects in the overall inflammatory response-related severe human diseases, including the cardiovascular ones. Moreover, cannabinoids have also appeared to protect against atherosclerosis in experimental conditions [40].

\section{The PNEI of Cardiovascular Diseases}

A decreased MLT production has been described either in myocardial infarction or in cerebral ischemia, at least in 
experimental conditions [41,42]. Moreover, the evidence of low MLT production has appeared to be associated with a poor prognosis and predict heart failure or death itself within 6 months after myocardial infarction [41]. Moreover, because the anti-inflammatory action of MLT, as well as its inhibitory action on the monocyte-macrophage system, the decline in MLT secretion could be at least in part responsible for cardiac ischemia-related decline in LMR (5), which may predict a poor prognosis, being the expression of an exaggerated inflammatory response. The evidence of a diminished MLT production has also been proven to predispose to hypertension [43].

On the same way, because of the neurotrophic action of MLT [34,44], its decline during the cerebral ischemia could compromise the tissue repairing mechanisms and explain the worse prognosis occurring in stroke patients in the presence of a marked decline in MLT blood concentrations. In any case, a concomitant evaluation of changes in LMR values and MLT secretion will be needed to establish whether cardiovascular disease-related activation of the monocyte-macrophage system with a following decline in LMR values may depend at least in part on a diminished pineal function, which in contrast may inhibit macrophage-mediated inflammatory response and activate $\mathrm{T}$ lymphocyte system, which plays an essential role in tissue repairing processes. Moreover, since tissue damage occurring in cerebral and myocardial ischemia may be enhanced by both IL-17 and IL-18, their inhibition could improve the prognosis of both stroke and myocardial infarction. More in detail, human heart tissue has been proven to contain pre-formed IL-18 in macrophage and endothelial cells.

The reduction of IL-18 activity with strategies such as the administration of anti-IL-18 monoclonal antibodies or its binding protein, the so-called IL-18BP, has been shown to reduce the impairment of the ischemia reperfusion injury in experimental conditions [26]. In addition, IL-18 blood concentrations have appeared to be significantly higher in patients who died from heart failure after myocardial infarction compared with survivors. Then, IL-18 could be responsible for myocardial infarction complications by activating the macrophage system, with a following amplification of the inflammatory response and a consequent enhanced tissue damage. Unfortunately, at present there is no study carried out to evaluate IL-18 blood levels in relation to changes occurring in the monocyte function and in LMR values during the clinical course of the cardiovascular diseases.

\section{New Therapeutic Implications}

According to the knowledgements available up to now, the possibility to modulate the cytokine network could have important therapeutic implications in the treatment of all severe systemic human diseases, including the cardiovascular ones. Then, the main problem becomes to establish which may be the optimal, the less toxic and less expensive way to act on the endogenous secretion of cytokines, which may be considered as hormonal-like substances within the immune system. From this point of view, because of their multiple and often contradictory and opposite activities, the possibility to cure inflammatory response-related systemic human diseases related to an altered immune response, including the cardiovascular ones, is a still only at the beginning, since most cytokines may correct some immune alterations, but on the same way induce other undesirable immunobiological events or systemic toxicities, with the only exception for IL-2, which represents up to now the only cytokine able to increase lymphocyte count and correct the severe lymphocytopenia occurring in cancer and other systemic diseases [3-5].

Therefore, based on the existence of a well-documented psycho-neuroendocrine regulation of the cytokine network, the optimal and most simple way to influence cytokine secretion could consisting of acting on the neuroendocrine modulation of the cytokine-mediated immune response. At present, the pineal hormones, the cannabinoid agents and the opioid antagonists have been already shown to be able to modulate the cytokine network. In more detail, MLT and other pineal indoles could be effective to reduce tissue damage induced by myocardial infarction and cerebral ischemia, whereas cannabinoid could be useful to counteract cancer and autoimmune diseases-related chronic exaggerated inflammatory response.

\section{References}

1. Lissoni P (2017) Therapy implications of the role of interleukin-2 in cancer. Exp Rev Clin Immunol 13(5): 491-498.

2. Zou W (2006) Regulatory $T$ cells, tumor immunity and immunotherapy. Nat Rev Immunol 6(4): 295-307.

3. Nishijma TE, Muss HB, Shachar SS, Tamura K, Takamatsu Y (2015) Prognostic value of lymphocyte-to-monocyte in patients with solid tumors: a systematic review and meta-analysis. Cancer Treat Rev 41: 971-978.

4. Du J, Chen, Shi J, Zhu X, Ying H, et al. (2017) The association between the lymphocyte to-monocyte ratio and disease activity in rheumatoid arthritis. Clin Rheumatol 36(12): 2689-2695.

5. Kurtul A, Yarlioqlues M, Celik E, Duran M, Elcik D, et al. (2015) Association of lymphocyte to-monocyte ratio with the no-reflow phenomenon in patients who underwent a primary percutaneous coronary intervention for ST-elevation myocardial infarction. Coron Arthery Dis 26(8): 706-712.

6. Riesco A (1970) Five-year cancer cure: relation to total amount of peripheral lymphocytes and neutrophils. Cancer 25: 135-140.

7. Grivennikov SI, Greten FR, Karin M (2010) Immunity, inflammation, and cancer. Cell 140(6): 883-899.

8. Grimm EA, Mazumder A, Zhang HZ, Rosenberg SA (1982) Lymphokineactivated killer cell phenomenon. Lysis of natural killer-resistant fresh solid tumor cells by interleukin 2-activated autologous human peripheral blood lymphocytes. J Exp Med 155(6): 1823-1841.

9. Antony PA, Restito NP (2005) CD4+CD25+ T regulatory cells, immunotherapy of cancer and interieukin-2. J Immunother 28(2): 120128.

10. Korn T, Bettelli E, Oukka M, Kuchroo VK (2009) IL-17 and Th17 cells. Annu Rev Immunol 27: 4854-517. 
11. Mills CD (2012) Ml and M2 macrophages: oracles of health and disease. Crit Rev Immunol 32(6): 463-468.

12. Mantovani A, Aliavena P, SicaA, Balkwiii E (2008) Cancer-related inflammation. Nature 454(7203): 436-444.

13. Prochazkiva J, Pokoma K, Holan V (2012) IL-12 inhibits the TGF-betadependent $\mathrm{T}$ celi development programs and skews the TGF-betainduced differentiation into Thl-like direction. Immunobiology 217(1): 74-82.

14. Nahrendorf M, Swirski FK (2016) Abandoning M1/M2 for a network model of macrophage function. Circulation Res 119(3): 414-417.

15. Lameijer MA, Tang J, Nahrendorf M, Beelen RHJ, Mulder WJM (2013) Monocytes and macrophages as nonmedicinal targets for improved diagnosis and treatment of disease. Expert Rev Mol Diagn 13(16): 567 580 .

16. Ldzkowska E, Eljasewicz A, Miklasz P, Musial WJ, Tycinska AM, et al. (2015) The role of different monocyte subsets in the pathogenesis of atherosclerosis and acute coronary syndromes. Scand J Immunol 82 163-173.

17. Fumagalli L, Lissoni P, Di Felice G, Meregalli S, Valsuani G, et al (1999) Pre-treatment serum markers and lymphocyte response to interleukin-2 therapy. Br J Cancer 80(3-4): 407-411.

18. Yang Y, Zhang L, Yu C, Vang XF (2014) Monocyte and macrophage differentiation : circulating inflammatory monocytes as biomarker for inflammatory diseases. Biomarker Res 2: 1-5.

19. Li F, Yang Y, Zhu X, Huang UL, Xu J (2015) Macrophage polarization modulates development of systemic lupus erythematosus. Cell Physiol Biochem 37(4): 1279-1288.

20. GaryT, Pichler M, Belaj K, Eller P, Hafner E, et al. (2014) Lymphocyteto-monocyte ratio: a novel marker for critical limb ischemia in PAOD patients. Int Clin Practice 68(12): 1483-1487.

21. Hanhua Ji, Yang Li, Zeyvan F, Bo Z, Xinwen i, et al. (2017) Monocyte/ lymphocyte ratio predicts the severity of coronary heart disease: a syntax score assessment. BCM Cardiovasc Dis 17(1): 90-97.

22. Brivio F, Fumagalli L, Parolini D, Messina G, Rovelli F, et al. (2008) T helper-to-T regulatory lymphocyte ratio as a new immunobiological index to quantify the anticancer immune status in cancer patients. In Vivo 22(5): 647-650.

23. Paramalingam SS, Thumboo J, Vasoo 5, Thio ST, Tse C, et al. (2007) In vivo pro- and anti-inflammatory cytokines in normal and patients with rheumatoid arthritis. Ann Acad Med Singapore 36(2): 96-99.

24. He J, Zhang X, Li Z (2016) Low-dose interieukin-2 treatment selectively modulates CD4 $\mathrm{T}$ cell subsets in patients with systemic lupus erythematosus. Nat Med 22(9): 991-993.

25. Hoeve MA, Savage ND, de Boer T, Langenberg DM, de Wall Malefyt R, et al. (2006) Divergent effects of IL-12 and IL-23 on the production of IL-17 by human T cells. Eur J Immunol 36(3): 661-670.

26. Dinarello CA, Kaplanski G (2005) interleukin-18 treatment options for inflammatory diseases. Exp Rev Clin immunol 1(4): 619-632.
27. Rubinow DR (1990) Brain, behaviour and immunity: an interactive system. J Natl Cancer Inst Monogr 10: 79-82.

28. Nagarkatti P, Pandey R, Rieder SA, Hegde VL, Nagarkatti M (2009) Cannabinoids as novel anti-inflammatory drugs. Future Med Chem 1(7): 1333-1349.

29. Conti A, Maestroni JGM (1995) The clinical immunotherapeutic role of melatonin in Oncology. J Pineal Res 19(3): 103-110.

30. Manfredi B, Sacerdote P, Bianchi M (1993) Evidence for an opioid inhibitory tone on T cell proliferation. J Neuroimmunol 44(1): 43-46.

31. Lewis JW, Shavit Y, Terman GV (1983) Apparent involvement of opioid peptides in stress-induced enhancement of tumor growth. Peptides 4(5): 635-638.

32. Lissoni P (1999) The pineal gland as a central regulator of cytokine network. Neuroendocrinol Lett 20(6): 343-349.

33. Ge D, Dauchi RT, Liu S, Zhang O, Mao L, et al. (2013) Insulin and IGF1 enhance lL-17-induced chemokine expression through a GSK3Bdependent mechanism: a new target for melatonin's anti-inflammatory action. J Pineal Res 55(4): 377-387.

34. Li Z, Li X, Chan MTV, Wu WKK, Tan D 0 Melatonin antagonizes interleukin-18-mediated inhibition of neural stem cell proliferation and differentiation. J Cell Mol Med 21(9): 2163-2171.

35. Bartsch C, Bartsch H (1999) Melatonin in cancer patients and in tumorbearing animals. Adv Exp Med Biol 467: 247-264.

36. Brugger P, Mrktl W, Herold M (1995) Impaired nocturnal secretion of melatonin in coronary heart disease. Lancet 345(8962): 1408.

37. Barjavel Mi, Mamdouh Z, Ragnbate N, Barouche O (1998) Differentiatial expression of the melatonin receptor in human monocytes. J Immunol 160(3): 1191-1197.

38. Sze SF, Ng TB, Liu WK (1993) Antiproliferative effect of pineal indoles on cultured tumor cell lines. J Pineal Res 14(1): 27-33.

39. Smith M, Lutgendorf SK, Sood AK (2010) Impact of stress on cancer patients. Future Oncol 6: 1683-1881.

40. Veiiiard NR, Arnaud C, Pelli G, Burger F, Staub C, et al. (2005) Low dose oral cannabinoid therapy reduces progression of atherosclerosis. Nature 434(7034): 782-783.

41. Dominguez-Rodriguez A, Abreu-Gonzales M, Reiter RJ (2006) Prognostic values of nocturnal melatonin levels as a novel marker in patients with ST-segment elevation myocardial infarction. Am J Cardiol 97(8): 1162-1164.

42. Pei Z, Pang SF, Cheung RT (2003) Administration of melatonin after onset of ischemia reduces the volume of cerebral infarction in rat middle cerebral artery occlusion stroke model. Stroke 34(3): 770-775.

43. Forman JP, Curhan GC, Schernammer ES (2004) Urinary melatonin and risk of incident hypertension among young women. J Hypertens 28(3): 446-451.

44. Brzezinski A (1997) Melatonin in humans. N Engl J Med 336(3): 186195. 

(C) (i) $\begin{aligned} & \text { This work is licensed under Creative } \\ & \text { Commons Attribution 4.0 Licens }\end{aligned}$ BY DOI: 10.19080/JETR.2019.05.555655

\section{Your next submission with Juniper Publishers} will reach you the below assets

- Quality Editorial service

- Swift Peer Review

- Reprints availability

- E-prints Service

- Manuscript Podcast for convenient understanding

- Global attainment for your research

- Manuscript accessibility in different formats ( Pdf, E-pub, Full Text, Audio)

- Unceasing customer service

Track the below URL for one-step submission https://juniperpublishers.com/online-submission.php 